\title{
Numerical Simulation of Aerodynamic Forces of ACSR Conductor
}

\author{
Lizhong Qi, Meng Wang ${ }^{\mathrm{a},{ }^{*}}$, Gang Xu, Zhangqi Wang \\ Department of Mechanical Engineering, North China Electric Power University, Baoding Hebei \\ 071003, China \\ aemail:wangmeng2146@163.com
}

Keywords: ACSR Conductor, aerodynamic characteristics, numerical simulation, roughness

\begin{abstract}
The aerodynamic characteristics of ACSR conductor with the large section were simulated with the fluid dynamics software in this paper. The slightly compressible viscous fluid and the Spalart-Allmaras turbulence model were used to modify the wind around the conductor. The numerical simulation results with the existing test results were compared and the effect of the roughness of the conductor on the aerodynamic characteristics was investigated. As the Reynolds number increases, the steady-state drag coefficients of the large section ACSR conductor decrease and tends to a stable value. The drag and lift coefficients decrease obviously in the range of 20000 to 40000 for the Reynolds number. The fluctuant lift coefficients of smooth boundary conductor are always larger than that of rough boundary model.
\end{abstract}

\section{Introduction}

Among the different mechanical vibration phenomena in high-voltage electrical overhead transmission lines, wind-excited oscillations of the conductors constitute a major issue. The most common of these vibrations are those generated by vortex shedding in the frequency range of approximately 2-50 Hz, corresponding to wind speeds of 1-10 m/s. Although such vibrations are barely perceptible due to their low amplitudes, they are, however, extremely important since they may lead to conductor fatigue at the positions of high-strain concentration. The determination of the aerodynamic coefficients of the conductors is necessary to estimate the vibration of overhead conductor and to ensure the reliability of the transmission lines [1].

At the beginning of the last century, a lot of research activities had been conducted and a series research results had been obtained in the 1990s [2-6]. In the research of the coupled fluid-structure vibration of overhead conductor, the force acting on the vibration objects were considered to be equivalent to the force that fluid with the same yaw angle and relative velocity acted on the same fixed objects, and dynamics equations and the criterion to judge the stable region of the galloping of the conductor were obtained. In the analysis of wind induced vibration of the conductor, the unsteady aerodynamic characteristics were related to the flow separation and the generation of the vortex and have strong nonlinear characteristics, so it was difficult to use simple theory or some analytical formula to describe the unsteady aerodynamic characteristics due to various actual conductors and the diversity and complexity of the iced conductors. Most of the early results were obtained by the wind tunnel experiment and established empirical formulae of the aerodynamic parameters.

In recent years, many scholars do a lot of research works in the field of disaster prevention of the transmission lines. The references [7-8] tested the static and dynamic aerodynamic characteristics of the galloping of iced bundled power lines. However, the wind tunnel test method, which is the important method to study the aerodynamic characteristics, can not meets the demand of diversity of the practical engineering due to their high cost, long testing cycle, measuring accuracy, limitations of methods, the equivalence problem of test models and practical problems. The development of the numerical wind tunnel technique based on computational fluid dynamics (CFD) provides a new way to calculate the fluid dynamics parameters of the conductor. The reference [9] analyzed the drag coefficients of the bundled conductor and found that the drag coefficients defined in the Chinese code might be over-estimated. The reference [10] studied the external flow field around transmission line 
under iced conditions and analyzed the distribution characteristics of velocity field, temperature field and pressure field of the airflow around transmission line. The reference [11] numerically simulated the aerodynamic characteristics of the iced power conductor and three bundled iced power transmission lines in different arrangement.

The aerodynamic characteristics of the large cross section conductor ( ACSR, Aluminium Cable Steel Reinforced ) were simulated with the fluid dynamics software in this paper. The slightly compressible viscous fluid and the turbulence modeled using large eddy simulation (LES) with the Spalart-Allmaras turbulence model. The numerical simulation results with the existing wind tunnel test results were compared and the effect of the roughness of the conductor on the aerodynamic characteristics was investigated for the engineering application of large cross section conductors.

\section{The aerodynamic force parameters and calculation model}

\subsection{The aerodynamic parameters of the conductor}

The pattern of air flow affects the magnitudes of wind loads exerting on the overhead transmission line. When the fluid flows through the object, the boundary layer separation point and the characteristics of vortex shedding are closely related to the Reynolds number, and the Reynolds number of the wind can be expressed as:

$$
R e=\frac{V d}{v}
$$

where, $V$ is the wind velocity, $\mathrm{m} / \mathrm{s}$; $d$ is the diameter of the conductor, $\mathrm{mm}$; $v$ is the air kinetic viscosity, $\mathrm{m}^{2} / \mathrm{s}$.

Generally, velocity in the rang of 0.5 to $10 \mathrm{~m} / \mathrm{s}$ can induce breeze vibration of the conductor, while velocity in the rang of $10 \sim 20 \mathrm{~m} / \mathrm{s}$ induce conductor dancing. Therefore the simulation wind velocities

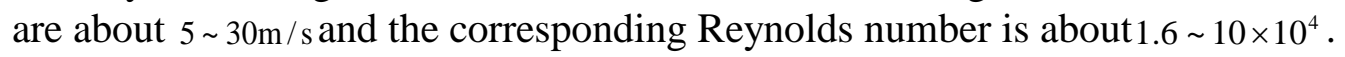

The drag force, lift force and moment force exerting on the conductor are related to the air density, wind velocity and the feature size of the conductor. In the process of vortex shedding, the fluctuant lift force $F_{\mathrm{L}}^{\prime}$ and the fluctuant moment force $M^{\prime}$ at a frequency of $f_{\mathrm{s}}$ periodically change their magnitudes and directions. The drag force consists of two parts: one is the steady-state drag force $F_{\mathrm{D} 0}$ which does not change with time; the other part is the fluctuant drag force $F_{\mathrm{D}}^{\prime}$ which changes its magnitude and direction at a frequency of $2 f_{s}$. The steady-state drag coefficients, fluctuant drag coefficients, fluctuant lift coefficients and fluctuant moment coefficients are defined as:

$$
\begin{aligned}
& C_{\mathrm{D} 0}=F_{\mathrm{D} 0} / 0.5 \rho d V^{2} \\
& C_{\mathrm{D}}^{\prime}=\overline{F_{\mathrm{D}}^{\prime}} / 0.5 \rho d V^{2} \\
& C_{\mathrm{L}}^{\prime}=\overline{F_{\mathrm{L}}^{\prime}} / 0.5 \rho d V^{2} \\
& C_{\mathrm{M}}^{\prime}=\overline{M^{\prime}} / 0.5 \rho d^{2} V^{2}
\end{aligned}
$$

Then the drag coefficients, the lift coefficients and the moment coefficients can be expressed as:

$$
\begin{aligned}
& C_{\mathrm{D}}=C_{\mathrm{D} 0}+C_{\mathrm{D}}^{\prime} \sin 2 f_{\mathrm{s}} t \\
& C_{\mathrm{L}}=C_{\mathrm{L}}^{\prime} \sin f_{\mathrm{s}} t \\
& C_{\mathrm{M}}=C_{\mathrm{M}}^{\prime} \sin f_{\mathrm{s}} t
\end{aligned}
$$

where $F_{\mathrm{D} 0}$ is the steady-state drag force of per unit conductor length , the positive direction is along the flow velocity; $\overline{F_{\mathrm{D}}^{\prime}}, \overline{F_{\mathrm{L}}^{\prime}}, \overline{M^{\prime}}$ are the amplitudes of the fluctuant drag force, the fluctuant lift force and the fluctuant moment force; $\rho$ is the air density; $V$ is the wind velocity; $d$ is the feature size, which is defined as the diameter of the conductor. 
The diameter of the conductor JL1/G3A-1250/70-76/ is $47.35 \mathrm{~mm}$, there are 28 outside aluminum strands with the diameter of $4.58 \mathrm{~mm}$. Figure 1 gives the model of a large cross section conductor, and the roughness of the conductor $R$ can be expressed as:

$$
R=\frac{d}{D}
$$

where, $d$ is the outside diameter of the conductor and $D$ is the diameter of outside strand . In this experiment the roughness of the large cross section conductor is 10.34 .

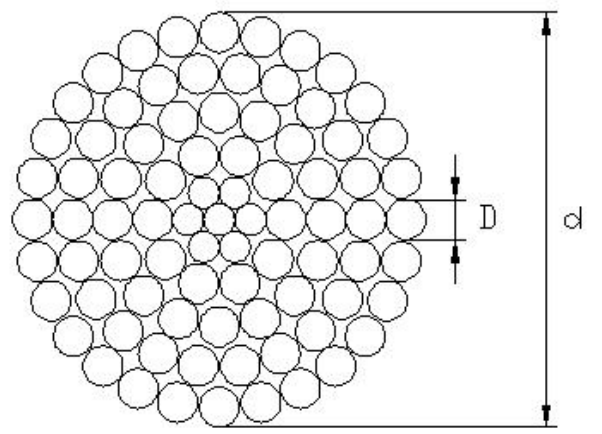

Figure 1: The model of a large cross section conductor.

\subsection{The external flow field model for the conductor}

Because the conductor belongs to a slender structure, the aerodynamic characteristics of the conductor mainly depend on the section shape, so two-dimensional simplified model can be used. The aerodynamic characteristics of the large cross section conductor are simulated through the FLUENT 12.0 software. The width of the flow field is set to be more than 10 times the diameter of the conductor when establishing the model of the external flow field. The diameter of the large cross section conductor is $47.35 \mathrm{~mm}$, and the computational domain is set to be the radius of the circular region which is $500 \mathrm{~mm}$.

For the discretization of the fluid domain, a block-structured grid is used. Block-structured grids, which are globally unstructured but locally structured, can be viewed as a compromise between the high geometrical flexibility of fully unstructured grids and the high numerical efficiency achieved on globally structured grids. The computational domain is meshed by the quadrilateral unstructured grid, then refines the region around the conductor. The mesh is shown in Figure 2.

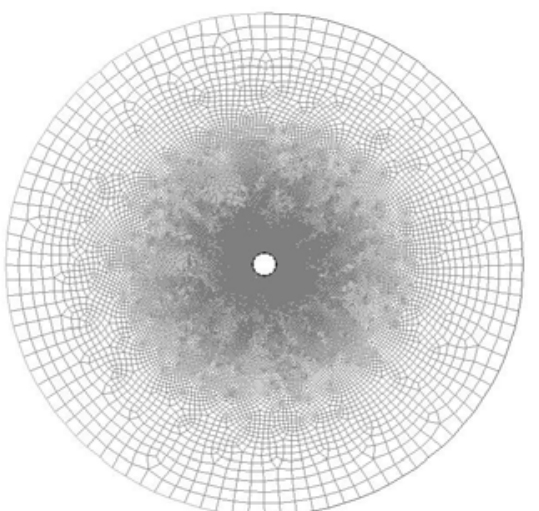

Figure 2: Mesh of the rough boundary model of the large cross section conductor.

The finite volume method is used for calculation and the Spalart-Allmaras turbulence model is selected. The left side of the computational domain is set to be a velocity-inlet boundary, the right side is set to be an outflow boundary, and the conductor is set to be a wall boundary. The air density is $1.205 \mathrm{~kg} / \mathrm{m}^{3}$, the air kinetic viscosity is $1.48 \times 10^{-5} \mathrm{~m}^{2} / \mathrm{s}$, and the turbulent viscosity ratio is 10 . In order to solve the velocity and pressure coupling problem in the momentum equations, this paper adopts the SIMPLEC algorithm. In order to overcome numerical stabilization for the high Reynolds number 
flows, quadratic up wind scheme (QUICK) is utilized, which is of third-order accuracy for the advection and remains only second-order for other terms such as diffusion ${ }^{[12]}$.

\section{The calculation results}

\subsection{Calculation}

The lift coefficient, drag coefficient and moment coefficient of the rough boundary model of the large cross section conductor at the speed of $5 \mathrm{~m} / \mathrm{s}$ is calculated. Figure 3 shows the iterative maps of the drag coefficient.

Lift force is produced in two forms: One is induced by the vortex shedding; the other is induced when the iced conductor produces asymmetric airfoil structure and assembles with the airflow. Because the conductor is a symmetrical structure, the average lift force induced by the vortex shedding is generally conceded to be zero, so the lift coefficients always fluctuate up and down as a sine wave around zero with a frequency equals to the vortex shedding frequency.

Drag force is induced by the skin-friction force and the pressure difference of the conductor when air flows through a conductor. The generation and release of the vortex also produce a drag force along the flow, which is also a periodic force with two maximum values and two minimum values per lift cycle.

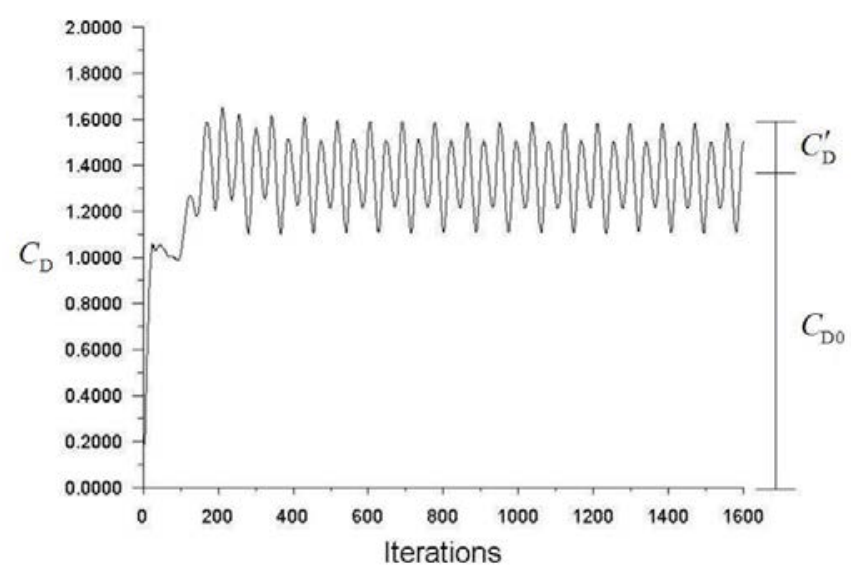

Figure 3: The iterative map of the drag coefficients at the speed of $5 \mathrm{~m} / \mathrm{s}$.

The frequency of the drag force is two times the vortex shedding frequency. The amplitude of fluctuant drag forces is different due to the asymmetry grid; here this paper adopts the larger as shown in Figure 3. For the cross section of conductors is of circular, the force arm of each point is same due to the symmetry of the structure, the moment coefficients are very small. The moment force changes periodically at a frequency of $f_{s}$, which is same as the changing frequency of left force of the conductor.

\subsection{Results}

Figure 4 showns the comparison of the aerodynamic characteristics of numerical simulation results with wind tunnel test results $[12,13]$, using rough and smooth boundary model of the large cross section power conductors under wind.

As can be seen from Figure 4:

i) The numerical simulation results substantially agree well with the test results, but there are obvious differences in the local characteristics: The steady-state drag coefficients of wind tunnel test decrease significantly in the Reynolds number range of 20000 to 40000, there is a rebound in the Reynolds number range of 40000 to 60000 and it gradually stabilizes at a Reynolds number above 60000. The steady-state drag coefficients of rough boundary model decrease significantly in the Reynolds number range of 20000 to 40000 and it gradually stabilizes at a Reynolds number above 40000. The steady-state drag coefficients of smooth boundary model decrease significantly in the Reynolds number range of 20000 to 60000 and it gradually stabilizes at a Reynolds number above 
60000. And the decrease of the test results is larger than that of the numerical simulation results; the steady-state drag coefficients of the numerical simulation are slightly larger than that of the test.

ii) The steady-state drag coefficients of smooth boundary model are larger than that of rough boundary model in the Reynolds number range of 20000 to 40000 . The rough boundary model achieves a near-stable value of 0.99 at a Reynolds number above 60000 , the wind tunnel test achieves a near-stable value of 0.94 and the smooth boundary model achieves a near-stable value of 0.91 . It can be seen that the larger the roughness $R$ is, the smaller the stable value is.

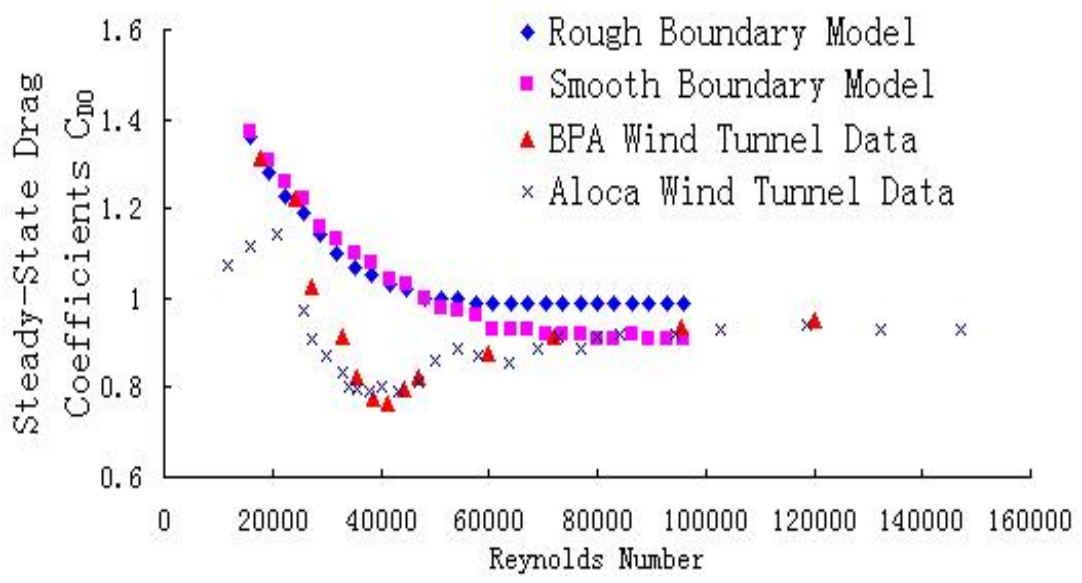

Figure 4: Comparison of the results obtained by numerical simulation with wind tunnel test results at different Reynolds number.

\section{Conclusions}

The aerodynamic characteristics of rough and smooth boundary model of the same power conductor are simulated through the FLUENT software and the following conclusions are obtained:

i) When the stable airflow blows the transmission conductor, there are vortexes generated in the leeward side of the conductor and the vortexes alternate and rotate in opposite directions. The lift coefficients always fluctuate up and down as a sine wave around zero at a frequency of $f_{s}$.

ii) As the velocity increases, a drop of the steady-state drag coefficients occurs in the Reynolds number range from 20000 to 40000, and the drag coefficients of the conductor increase with the velocity reduces.

iii) The steady-state drag coefficients of smooth boundary model are slightly higher than that of rough boundary model in the Reynolds number range from 20000 to 40000, both rough and smooth boundary model achieve a stable value at a Reynolds number above 60000.

\section{References}

[1] A.L. Braun, A.M. Awruch. Aerodynamic and aeroelastic analysis of bundled cables by numerical simulation, Journal of Sound and Vibration, Vol. 284 (2005), p. 51-73.

[2] M. I. Kazakevich, A. G. Vasilenko. Closed analytical solution for galloping aeroelastic self-oscillations, Journal of Wind Engineering and Industrial Aerodynamics, Volume 65, Issues 1-3, Pages 353-360 (December 1996).

[3] Nigol, O. ;Buchan, P. G. Conductor Galloping Part I-Den Hartog Mechanism. Power Apparatus and Systems, IEEE Transactions on, Volume : PAS-100 , Issue: 2 : 1981 , Page(s): 699 - 707.

[4] Nigol, O. ; Buchan, P.G. Conductor Galloping-Part II Torsional Mechanism. Power Apparatus and Systems, IEEE Transactions on. Volume : PAS-100, Issue: 2 1981, Page(s): 708 - 720.

[5] P. Parkinson and N. P. H. Brooks, On the aeroelastic instability of bluff cylinders, Journal of Applied Mechanics, vol. 28, pp. 252-258, 1961. 
[6] P. Yu, Y. M. Desai, A. H. Shah, and N. Popplewell, Three-degree-of-freedom model for galloping. Part 1: formulation, Journal of Engineering Mechanics, vol. 119, no. 12, pp. 2404-2425, 1993.

[7] LI Wangping, YANG Xinxiang, ZHANG Lizhi. Static aerodynamic characteristics of the galloping of bundled iced power transmission lines [J]. Acta Aerodynamica Sinica, 1995,13(4):427-433.

[8] LI Wangping. Dynamic aerodynamic characteristics of the galloping of bundled iced power transmission lines [J]. Acta Aerodynamica Sinica, 2000, 18(4):413-420.

[9] Cai Mengqi, YAN Bo, LIU Xiaohui, et al. Analysis on drag coefficients of bundled conductors under wind load [J]. Journal of Chongqing University, 2013, 36(1):110-114.

[10] HUAN XINbo, LIN Shufan, ZHU Yongcan, et al. Numerical Simulation and Analysis of External Flow Field around Transmission Line under Icing Condition [J]. Electric Power Construction, 2014, 35(5):6-12.

[11] LV Yi. Numerical Simulations of Aerodynamic Characteristics of Iced Conductors [D]. Zhejiang University, 2008.

[12] LV Yi, LOU Wenjuan, SUN Zhennmao, et al. Numerical simulation of aerodynamic characteristics of three bundled iced transmission lines [J]. Journal of Zhejiang University: Engineering Science, 2010, 44(1):174-179.

[13] R. L. Wardlaw, and K.R. Cooper, A Wind Tunnel Investigation of the Steady Aerodynamics Forces on Smooth and Stranded Twin Bundled Power Conductors for the Aluminum Company of America, LTR-LA-117, National Aeronautical Establishment, National Research Council Canada, 1973. 